\title{
The critical state behaviour of barodesy compared with the Matsuoka-Nakai failure criterion
}

\author{
Wolfgang Fellin* and Alexander Ostermann ${ }^{\dagger}$
}

3 Sept 2011

post-print of:

Fellin, W.; Ostermann, A. (2013): The critical state behaviour of barodesy compared with the Matsuoka-Nakai failure criterion. In: International Journal for Numerical and Analytical Methods in Geomechanics 37, pp. 299 - 308.

\begin{abstract}
Barodesy is a new approach to constitutive modelling of soil. It is based on Goldscheider's principles and maps stretching directions onto corresponding stress directions with the help of a simple exponential function. This mapping also determines a critical state surface in principal stress space. The paper investigates this surface and relates it to the well-known MatsuokaNakai failure criterion. It turns out that the difference between these two surfaces is negligible for practical applications.
\end{abstract}

\section{Introduction}

Under large shear deformations granular materials reach a critical state where stress and volume remain constant, see e.g. [9]. Barodesy [6], a new hypoplastic [4] approach, uses a simple exponential function that maps stretching directions onto stress directions. In particular, isochoric stretching is mapped to a critical state surface in the principal stress space. The Matsuoka-Nakai failure criterion

\footnotetext{
${ }^{*}$ Division of Geotechnical and Tunnel Engineering, University of Innsbruck, Austria
}

${ }^{\dagger}$ Department of Mathematics, University of Innsbruck, Austria 
[8] defines a surface in the principal stress space as well. The present paper aims at exploiting the mathematical structure of barodesy to investigate the similarity between these two surfaces. It is shown that their difference is negligible for practical applications.

\section{A short outline of barodesy}

As barodesy is quite a new approach to model the behaviour of cohesionless soil, we start with a short outline of this theory. Barodesy is based on two rules of Goldscheider [3] that refer to proportional paths ${ }^{1}$ applied to dry sand:

(i) Proportional strain paths $(P \varepsilon P)$ starting from the stress $\mathbf{T}=\mathbf{0}$ are associated to proportional stress paths $(P \sigma P)$.

(ii) Proportional strain paths starting from $\mathbf{T} \neq \mathbf{0}$ lead asymptotically to the corresponding proportional stress paths obtained when starting at $\mathbf{T}=\mathbf{0}$.

The main concept of barodesy was already published in [5], especially the implementation of the first rule which is studied here in detail. The model of the second rule has slightly changed since then, an actual version is resumed below.

The first rule is modelled by means of a function that relates the stretching $\mathbf{D}$ to a tensor $\mathbf{R}$ pointing in direction of the corresponding proportional stress path [5]

$$
\mathbf{R}(\mathbf{D})=\left(\operatorname{tr} \mathbf{D}^{0}\right) \mathbf{I}+c_{1} \exp \left(c_{2} \mathbf{D}^{0}\right) .
$$

The stretching $\mathbf{D}$ is the symmetric part of the velocity gradient, and $c_{1}, c_{2}$ are material constants. Here, the superscript 0 denotes the normalisation of a tensor $\mathbf{X}$, i.e. $\mathbf{X}^{0}=\mathbf{X} /|\mathbf{X}|$ with $|\mathbf{X}|=\sqrt{\operatorname{tr} \mathbf{X}^{2}}$.

The second rule translates into an evolution equation involving the Jaumann objective stress rate

$$
\stackrel{\circ}{\mathbf{T}}=\hat{f} \mathbf{R}^{0}+\hat{g} \mathbf{T} .
$$

According to [6] the functions $\hat{f}$ and $\hat{g}$ are chosen as

$$
\begin{aligned}
& \hat{f}=f \cdot \dot{\varepsilon} \cdot h(\sigma) \\
& \hat{g}=g \cdot \dot{\varepsilon} \cdot h(\sigma) / \sigma
\end{aligned}
$$

\footnotetext{
${ }^{1}$ Proportional stress and strain paths are characterised by constant ratios of the principal values $T_{1}: T_{2}: T_{3}$ and $\varepsilon_{1}: \varepsilon_{2}: \varepsilon_{3}$, respectively.
} 
Table 1: Material parameters of barodesy for Hostun RF sand, from [6].

\begin{tabular}{cccccc}
\hline$e_{c_{0}}$ & $c_{1}$ & $c_{2}$ & $c_{3}$ & $c_{4}$ & $c_{5}$ \\
\hline 1.0 & -1.7637 & -1.0246 & $2358 \mathrm{kPa}^{1-c_{4}}$ & 0.5519 & 0.5080 \\
\hline
\end{tabular}

with $\sigma=|\mathbf{T}|$ and $\dot{\varepsilon}=|\mathbf{D}|$. By the above choice, homogeneity of degree one with respect to $\mathbf{D}$ is preserved. From (2), (3) we get

$$
\left.\stackrel{\circ}{\mathbf{T}}=h(\sigma) \cdot\left[f \mathbf{R}^{0}+g \mathbf{T}^{0}\right)\right] \cdot \dot{\varepsilon} .
$$

This evolution equation enforces $\mathbf{T}^{0}$ to approach $\mathbf{R}^{0}$ for constant stretching, i.e. the stress direction $\mathbf{R}^{0}$ acts as attractor.

The function $h(\sigma)$ is set to

$$
h(\sigma)=c_{3} \sigma^{c_{4}}
$$

with $c_{4}<1$ to take barotropy into account. The critical state concept is implemented by assuming $f=f(e)$ and $g=g(\sigma)$. In [6], the following choice is proposed among others:

$$
\begin{array}{r}
f(e)=\frac{1+e_{c_{0}}}{1+e}-c_{5} \operatorname{tr} \mathbf{D}^{0} \\
g(\sigma)=-\exp \left(\frac{\sigma^{1-c_{4}}}{c_{3} c_{5}\left(1-c_{4}\right)}\right),
\end{array}
$$

with $e$ denoting the void ratio, and $e_{c_{0}}$ the critical void ratio at zero pressure. This version was also used in [2].

When the stress reaches the critical state, the stress rate vanishes, i.e. $\stackrel{\circ}{\mathbf{T}}=\mathbf{0}$, and the stress direction meets the direction of $\mathbf{R}$, i.e. $\mathbf{R}^{0}=\mathbf{T}^{0}$. It then follows from (4) that

$$
f(e)+g(\sigma)=0
$$

for critical states. This is the equation of the critical state line.

The material parameters for Hostun RF sand [1] are given in Tab. 1. They are taken from [6]. Exemplary numerical results of element tests are shown in Figs. 1 and 2 . 


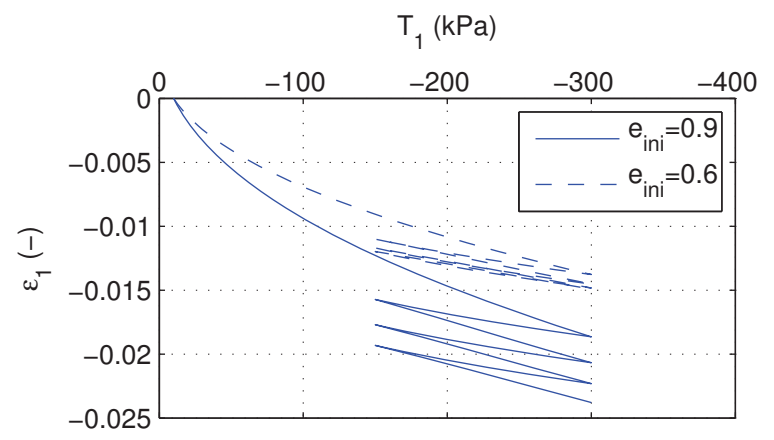

Figure 1: Oedometric compression: initial stress $T_{1}=-10 \mathrm{kPa}, T_{2}=T_{3}=K_{0} T_{1}$ with $K_{0}=R_{2} / R_{1}=0.4672$; initial void ratio $e_{\text {ini }}=0.6$, to represent a dense specimen and $e_{\mathrm{ini}}=0.9$, to represent a loose specimen; material parameters from Tab. 1.
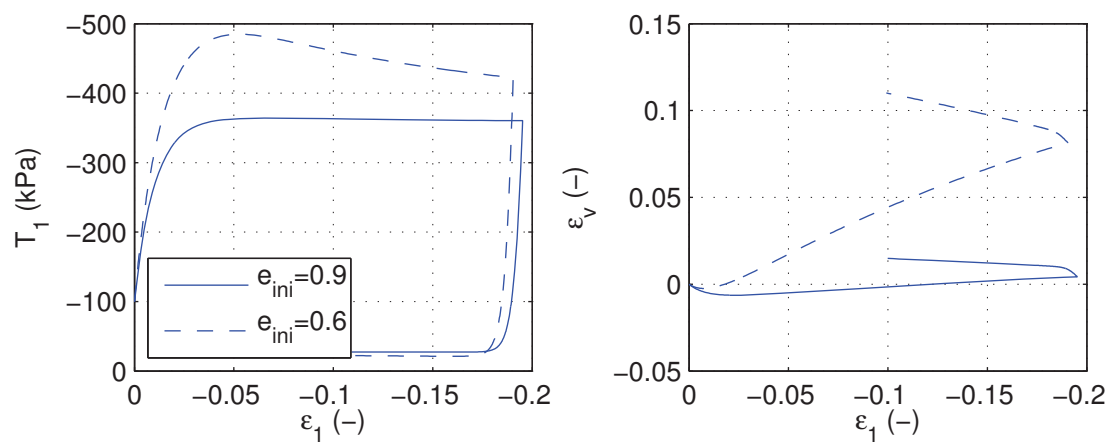

Figure 2: Triaxial compression: initial stress $T_{1}=T_{2}=T_{3}=-100 \mathrm{kPa}$; initial void ratio $e_{\text {ini }}=0.6$, to represent a dense specimen and $e_{\text {ini }}=0.9$, to represent a loose specimen; material parameters from Tab. 1. 


\section{The behaviour of barodesy for isochoric deforma- tions}

The mapping function $\mathbf{R}(\mathbf{D})$ constitutes the backbone of barodesy. It plays a key role in the critical state behaviour of the material model.

\subsection{Behaviour of the mapping function}

All stress directions $\mathbf{R}^{0}$ for isochoric stretching $(\operatorname{tr} \mathbf{D}=0)$ form a fan in the principal stress space, see Fig. 3. This fan is only determined by the material parameter $c_{2}$, due to the scaling with $|\mathbf{R}|$. Stress directions for compressive stretching are located inside this fan, those for expansive stretching outside, see Fig. 4.
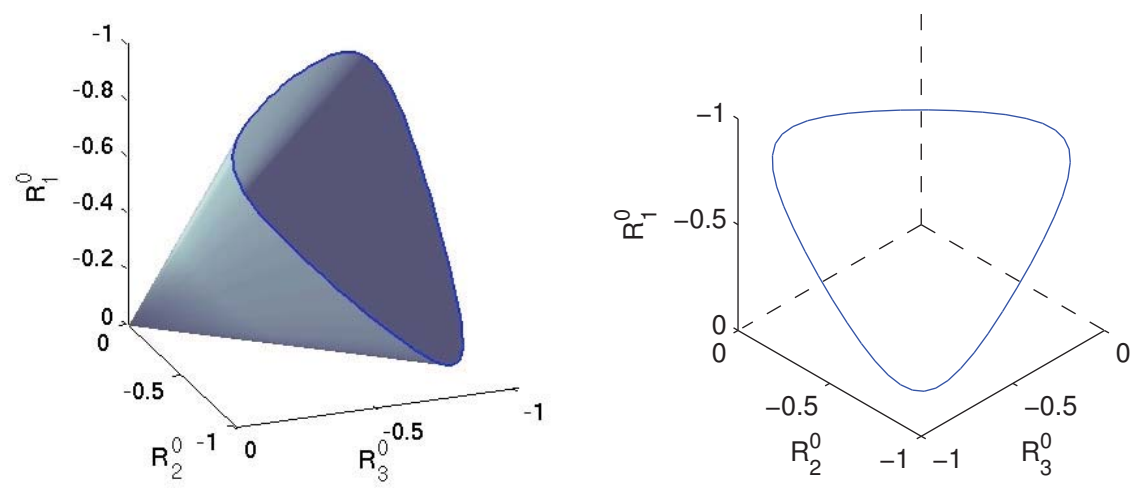

Figure 3: Fan of stress directions $\mathbf{R}^{0}$ for isochoric stretching $(\operatorname{tr} \mathbf{D}=0)$ in the principal stress space: surface representation (left), cross section with the deviatoric plane satisfying $\operatorname{tr} \mathbf{R}^{0} / \sqrt{3}=-1$ (right); $\mathbf{R}^{0}=\mathbf{R} /|\mathbf{R}|$ with $\mathbf{R}$ from (1), material parameters from Tab. 1.

Note that we have $\mathbf{R}^{0}=\mathbf{T}^{0}$ in (4) if a stress state is on a proportional stress path corresponding to the applied stretching. In that situation the stress rate $\stackrel{\circ}{\mathbf{T}}$ will $^{\circ}$ point in direction of the stress path as intended to obey Goldscheider's first rule, and $\mathbf{T}^{0}$ points in that direction, as well as $\mathbf{R}^{0}$ does. Therefore, the fan consisting of all directions $\mathbf{R}^{0}$ generated by isochoric stretching (Fig. 3) is equal to that formed by the proportional stress paths for isochoric strain paths. The latter acts as critical state surface and can therefore be compared with classical failure surfaces 

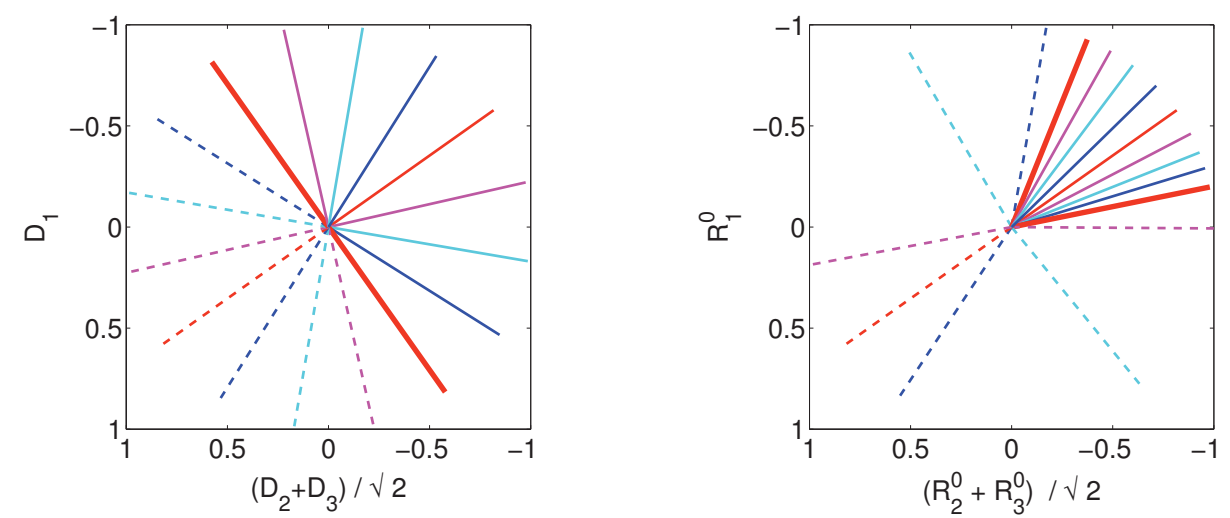

Figure 4: Stretching of proportional strain paths and corresponding stress directions $\mathbf{R}^{0}$ from mapping function (1) with material parameters of Tab. 1. Thick solid lines represent $\operatorname{tr} \mathbf{D}=0$, thin solid lines compression $(\operatorname{tr} \mathbf{D}<0)$ and thin dashed lines extension ( $\operatorname{tr} \mathbf{D}>0)$.

obtained for a critical friction angle. As both fans are identical, the first one can be compared with failure surfaces as well, without integrating the constitutive model.

\subsection{Relation to the critical state friction angle}

For states with $\stackrel{\circ}{\mathbf{T}}=\mathbf{0}$ it follows from (4) that $\mathbf{T}$ is proportional to $\mathbf{R}$ and thus $\mathbf{R}^{0}=\mathbf{T}^{0}$. For critical states, we have tr $\mathbf{D}=0$ and (1) simplifies to $\mathbf{R}(\mathbf{D})=$ $c_{1} \exp \left(c_{2} \mathbf{D}^{0}\right)$.

The ratio of the principal stresses in a triaxial stress state is $K_{c}=T_{3} / T_{1}=$ $T_{2} / T_{1}$. For critical states in cohesionless soils this can be written as

$$
K_{c}=\frac{1-\sin \varphi_{c}}{1+\sin \varphi_{c}}
$$

with the critical state friction angle $\varphi_{c}$. From $\mathbf{R}^{0}=\mathbf{T}^{0}$ it follows that $R_{3} / R_{1}=$ $T_{3} / T_{1}$. Further using $D_{1}=-1$ and $D_{2}=D_{3}=\frac{1}{2}$, we deduce

$$
K_{c}=\frac{R_{3}}{R_{1}}=\exp \left(c_{2}\left(D_{3}^{0}-D_{1}^{0}\right)\right)=\exp \left(\sqrt{\frac{3}{2}} c_{2}\right)
$$


Consequently, the material parameter $c_{2}$ is given by

$$
c_{2}=\sqrt{\frac{2}{3}} \ln K_{c}=\sqrt{\frac{2}{3}} \ln \left(\frac{1-\sin \varphi_{c}}{1+\sin \varphi_{c}}\right) .
$$

For $\varphi_{c}=33.8^{\circ}$ this yields $c_{2}=-1.0246$, compare Tab. 1 .

\section{Comparison with the Matsuoka-Nakai failure cri- terion}

The Matsuoka-Nakai failure criterion is a widely accepted approach for modelling limit stress states in granular material. It is implemented, e.g., in some of the actual versions of hypoplasticity $[11,10,7]$. We will compare this criterion with the behaviour of the $\mathbf{R}$-function.

\subsection{Matsuoka-Nakai failure criterion}

Matsuoka and Nakai [8] proposed the following failure criterion for cohesionless soils:

$$
\frac{I_{1} I_{2}}{I_{3}}=K_{\mathrm{MN}}
$$

with $I_{1}=\operatorname{tr} \mathbf{T}, I_{2}=\left(I_{1}^{2}-\operatorname{tr} \mathbf{T}^{2}\right) / 2, I_{3}=\operatorname{det} \mathbf{T}$ being the first, second and third invariant of the stress tensor, respectively. This criterion reads for principal stresses

$$
\frac{\left(T_{1}+T_{2}+T_{3}\right)\left(T_{1} T_{2}+T_{2} T_{3}+T_{3} T_{1}\right)}{T_{1} T_{2} T_{3}}=K_{\mathrm{MN}} .
$$

The material parameter $K_{\mathrm{MN}}$ can be expressed by the critical state friction angle

$$
K_{\mathrm{MN}}=\left(1+2 K_{c}\right)\left(1+\frac{2}{K_{c}}\right)=\frac{9-\sin ^{2} \varphi_{c}}{1-\sin ^{2} \varphi_{c}} .
$$

The cross section of (10) with a deviatoric plane in principal stress space looks quite similar to a cross section of the fan of stress directions $\mathbf{R}^{0}$ for isochoric stretching, compare Fig. 5 and Fig. 3. 


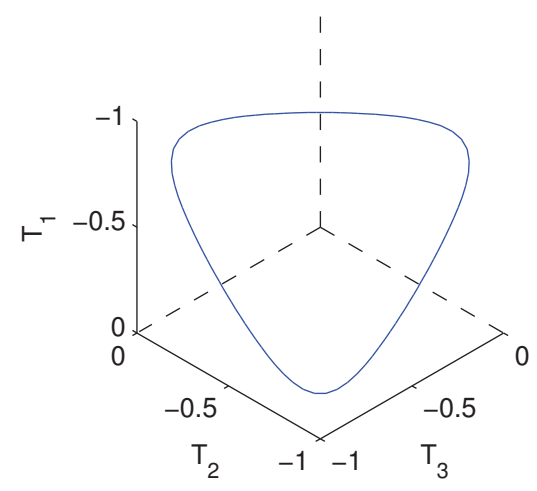

Figure 5: Cross section of the critical stresses satisfying the Matsuoka-Nakai failure criterion with the deviatoric plane satisfying $\operatorname{tr} \mathbf{T} / \sqrt{3}=-1, \varphi_{c}=33.8^{\circ}$.

\subsection{Numerical tests}

We can calculate the fan of the proportional stress paths for isochoric strain paths using the stretching

$$
\begin{aligned}
& D_{1}=\sqrt{\frac{2}{3}} \sin \left(\theta+\frac{2 \pi}{3}\right) \\
& D_{2}=\sqrt{\frac{2}{3}} \sin \theta \\
& D_{3}=\sqrt{\frac{2}{3}} \sin \left(\theta-\frac{2 \pi}{3}\right),
\end{aligned}
$$

for which $\operatorname{tr} \mathbf{D}=0$ and $|\mathbf{D}|=1$. Evaluating (1) for $0 \leq \theta \leq 2 \pi$ yields all directions $\mathbf{R}(\theta)$ of the required proportional stress paths, see Fig. 3. This fan is equal to that of the proportional stress paths due to the construction of barodesy (4).

A cross section of $\mathbf{R}(\theta)$ with a deviatoric plane results in stress states for which we can evaluate the left-hand side of the Matsuoka-Nakai criterion (10), namely

$$
K_{\mathrm{R}}(\theta)=\frac{I_{1}^{\mathrm{R}} I_{2}^{\mathrm{R}}}{I_{3}^{\mathrm{R}}}
$$

For $\varphi_{c}=33.8^{\circ}$ the variation of $K_{\mathrm{R}}(\theta)$ compared to the constant value of $K_{\mathrm{MN}}=$ 


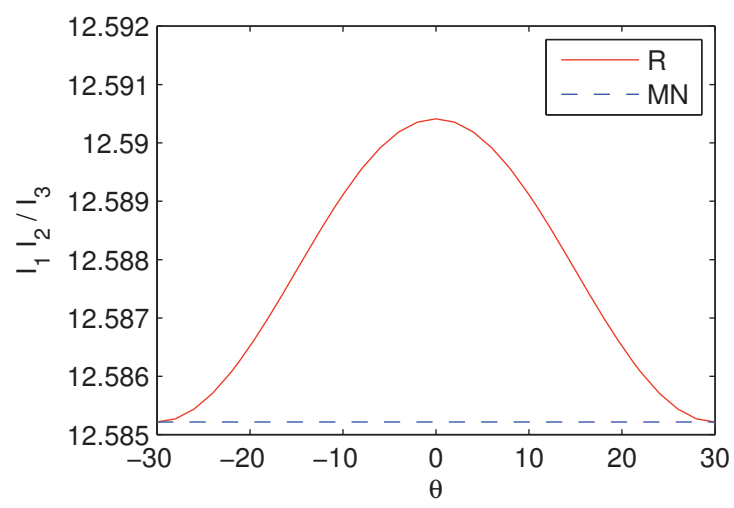

Figure 6: Left-hand side of the Matsuoka-Nakai criterion (10) evaluated with $\mathbf{R}(\theta)$.

12.5852 is displayed in Fig. 6. The angle $\theta$ is the so called Lode angle

$$
\theta=\frac{1}{3} \arcsin \left(\frac{-3 \sqrt{6} J_{3}}{\left|\mathbf{T}^{*}\right|^{3}}\right)
$$

with the deviatoric stress $\mathbf{T}^{*}=\mathbf{T}-\mathbf{I}(\operatorname{tr} \mathbf{T}) / 3$ and the third invariant of the deviatoric stress $J_{3}=\operatorname{det} \mathbf{T}^{*}$. A value of $\theta=-30^{\circ}$ corresponds to triaxial extension, $\theta=+30^{\circ}$ to triaxial compression. We see in Fig. 6 that $K_{\mathrm{MN}}=K_{\mathrm{R}}$ for triaxial stress states and only a small difference in between. The maximum value of $K_{\mathrm{R}}(\theta)$ for $\varphi_{c}=33.80^{\circ}$ is $K_{\mathrm{R}, \max }=12.5904$. We can calculate the friction angle for a Matsuoka-Nakai surface defined by this maximum by using (12)

$$
\varphi_{c}^{\mathrm{R}}=\arcsin \sqrt{\frac{K_{\mathrm{R}, \max }-9}{K_{\mathrm{R}, \max }-1}} .
$$

This yields $\varphi_{c}^{\mathrm{R}}=33.82$. The relative deviation

$$
\Delta \varphi_{c}=\frac{\varphi_{c}^{\mathrm{R}}-\varphi_{c}}{\varphi_{c}}
$$

for various $\varphi_{c}$ is plotted in Fig. 7. This relative difference is less than $0.12 \%$ for the range $20^{\circ} \leq \varphi_{c} \leq 40^{\circ}$. 


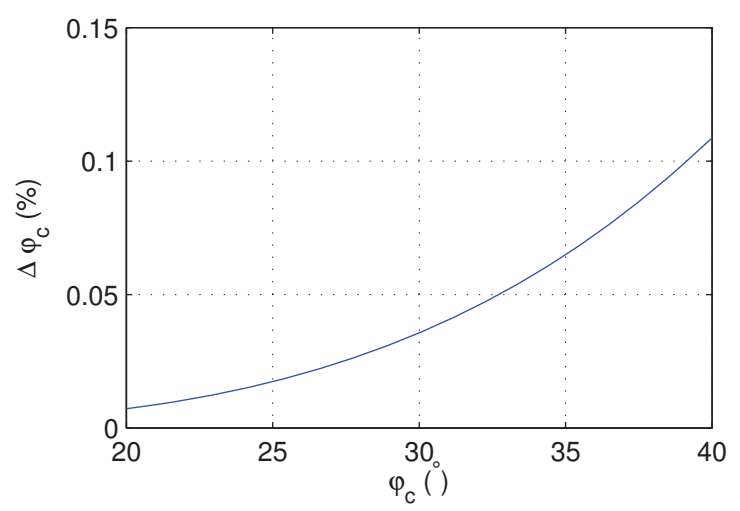

Figure 7: Maximum relative deviation of the $\mathbf{R}$-fan from the Matsuoka-Nakai failure surface.

\subsection{Mathematical analysis}

The aim of this section is to study the difference $K_{\mathrm{R}}(\theta)-K_{\mathrm{MN}}$ analytically. Remember that for constant stretching the direction $\mathbf{R}^{0}$ is an attractor for the stress, i.e. the evolution equation of barodesy (4) enforces $\mathbf{T}^{0}$ to approach $\mathbf{R}^{0}$. In the special case of isochoric stretching barodesy reaches the critical state stress, which then can be written as

$$
\mathbf{T}=\alpha \mathbf{R}
$$

where $\alpha$ denotes a given scalar function.

Consequently, using (1) we consider a stress tensor with components

$$
T_{j}^{\mathrm{R}}=\alpha R_{j}=\alpha c_{1} \mathrm{e}^{c_{2} D_{j}^{0}}, \quad j=1,2,3,
$$

defined by an isochoric stretching

$$
D_{1}^{0}=a \sqrt{\frac{2}{3}}, \quad D_{2}^{0}=b \sqrt{\frac{2}{3}}, \quad D_{3}^{0}=c \sqrt{\frac{2}{3}},
$$

where due to (13)

$$
\begin{aligned}
& a=a(\theta)=\sin \left(\theta+\frac{2 \pi}{3}\right)=-\frac{1}{2} \sin \theta+\frac{\sqrt{3}}{2} \cos \theta \\
& b=b(\theta)=\sin \theta \\
& c=c(\theta)=\sin \left(\theta-\frac{2 \pi}{3}\right)=-\frac{1}{2} \sin \theta-\frac{\sqrt{3}}{2} \cos \theta .
\end{aligned}
$$


From (14) and (18), we obtain by elementary calculations

$$
\begin{aligned}
K_{\mathrm{R}}(\theta) & =\frac{\left(T_{1}^{\mathrm{R}}+T_{2}^{\mathrm{R}}+T_{3}^{\mathrm{R}}\right)\left(T_{1}^{\mathrm{R}} T_{2}^{\mathrm{R}}+T_{2}^{\mathrm{R}} T_{3}^{\mathrm{R}}+T_{3}^{\mathrm{R}} T_{1}^{\mathrm{R}}\right)}{T_{1}^{\mathrm{R}} T_{2}^{\mathrm{R}} T_{3}^{\mathrm{R}}} \\
& =\left(T_{1}^{\mathrm{R}}+T_{2}^{\mathrm{R}}+T_{3}^{\mathrm{R}}\right)\left(\frac{1}{T_{1}^{\mathrm{R}}}+\frac{1}{T_{2}^{\mathrm{R}}}+\frac{1}{T_{3}^{\mathrm{R}}}\right) \\
& =3+2\left(\cosh \left(c_{2}\left(D_{1}^{0}-D_{2}^{0}\right)\right)+\cosh \left(c_{2}\left(D_{2}^{0}-D_{3}^{0}\right)\right)+\cosh \left(c_{2}\left(D_{3}^{0}-D_{1}^{0}\right)\right)\right) .
\end{aligned}
$$

Setting

$$
\kappa=c_{2} \sqrt{\frac{2}{3}}=\frac{2}{3} \ln K_{c}
$$

we finally get

$$
K_{\mathrm{R}}(\theta)=3+2(\cosh (\kappa(a-b))+\cosh (\kappa(b-c))+\cosh (\kappa(c-a))),
$$

where

$$
\begin{aligned}
& a-b=\sqrt{3} \sin \left(\frac{\pi}{6}-\theta\right) \\
& b-c=\sqrt{3} \sin \left(\frac{\pi}{6}+\theta\right) \\
& c-a=-\sqrt{3} \cos \theta .
\end{aligned}
$$

We first note that

$$
K_{\mathrm{R}}\left(\theta+\frac{\pi}{3}\right)=K_{\mathrm{R}}(\theta),
$$

i.e. $K_{\mathrm{R}}(\theta)$ is $\frac{\pi}{3}$-periodic. This is verified by inserting the relations

$$
\begin{aligned}
& a\left(\theta+\frac{\pi}{3}\right)=\sin (\theta+\pi)=-\sin \theta=-b(\theta) \\
& b\left(\theta+\frac{\pi}{3}\right)=\sin \left(\theta+\frac{\pi}{3}\right)=-\sin \left(\theta-\frac{2 \pi}{3}\right)=-c(\theta) \\
& c\left(\theta+\frac{\pi}{3}\right)=\sin \left(\theta-\frac{\pi}{3}\right)=-\sin \left(\theta+\frac{2 \pi}{3}\right)=-a(\theta) .
\end{aligned}
$$

into (20). Moreover, we have the symmetry

$$
K_{\mathrm{R}}\left(\frac{\pi}{3}-\theta\right)=K_{\mathrm{R}}(\theta), \quad 0 \leq \theta \leq \frac{\pi}{6}
$$


which follows from

$$
\begin{aligned}
& a\left(\frac{\pi}{3}-\theta\right)=\sin (-\theta+\pi)=\sin \theta=b(\theta) \\
& b\left(\frac{\pi}{3}-\theta\right)=\sin \left(\frac{\pi}{3}-\theta\right)=\sin \left(\theta+\frac{2 \pi}{3}\right)=a(\theta) \\
& c\left(\frac{\pi}{3}-\theta\right)=\sin \left(-\theta-\frac{\pi}{3}\right)=\sin \left(\theta-\frac{2 \pi}{3}\right)=c(\theta) .
\end{aligned}
$$

Therefore, it is sufficient to study the function $K_{\mathrm{R}}(\theta)$ on the interval

$$
0 \leq \theta \leq \frac{\pi}{6}
$$

To show that $K_{\mathrm{R}}(\theta)$ is (strictly) monotonically decreasing on $0 \leq \theta \leq \pi / 6$, we use the Taylor series expansion of the hyperbolic cosine

$$
\cosh z=1+\frac{z^{2}}{2 !}+\frac{z^{4}}{4 !}+\frac{z^{6}}{6 !}+\ldots
$$

in (20) and treat all powers separately. Inserting (21), one easily verifies that

$$
\begin{aligned}
& (a-b)^{2}+(b-c)^{2}+(c-a)^{2}=\frac{9}{2} \\
& (a-b)^{4}+(b-c)^{4}+(c-a)^{4}=\frac{81}{8}
\end{aligned}
$$

and that the higher powers

$$
(a-b)^{2 k}+(b-c)^{2 k}+(c-a)^{2 k}, \quad k \geq 3
$$

are strictly monotonically decreasing for $0 \leq \theta \leq \pi / 6$.

From these properties, we infer the following result: The function $K_{\mathrm{R}}(\theta)$ has its (global) maxima at $\theta=k \frac{\pi}{3}$ (for $k \in \mathbb{Z}$ ) and its (global) minima at $\theta=\frac{\pi}{6}+k \frac{\pi}{3}$ (for $k \in \mathbb{Z}$ ).

Setting $\theta=\frac{\pi}{6}$, we get

$$
a=b=\frac{1}{2}, \quad c=-1
$$


and thus

$$
\begin{aligned}
K_{\mathrm{R}}\left(\frac{\pi}{6}\right) & =5+4 \cosh \ln \left(\frac{1-\sin \varphi_{c}}{1+\sin \varphi_{c}}\right) \\
& =5+2\left(\frac{1-\sin \varphi_{c}}{1+\sin \varphi_{c}}+\frac{1+\sin \varphi_{c}}{1-\sin \varphi_{c}}\right) \\
& =5+\frac{4+4 \sin ^{2} \varphi_{c}}{1-\sin ^{2} \varphi_{c}} \\
& =\frac{9-\sin ^{2} \varphi_{c}}{1-\sin ^{2} \varphi_{c}} \\
& =K_{\mathrm{MN}} .
\end{aligned}
$$

This shows that the minima of $K_{\mathrm{R}}(\theta)$ coincide with $K_{\mathrm{MN}}$, compare Fig. 6 .

On the other hand, for $\theta=0$ we have

$$
a(0)=-c(0)=\frac{\sqrt{3}}{2}, \quad b(0)=0 .
$$

The maxima $K_{\mathrm{R} \text {,max }}$ of $K_{\mathrm{R}}(\theta)$ are thus given by

$$
K_{\mathrm{R}, \max }=K_{\mathrm{R}}(0)=3+4 \cosh \left(\frac{\sqrt{3}}{3} \ln K_{c}\right)+2 \cosh \left(\frac{2 \sqrt{3}}{3} \ln K_{c}\right) .
$$

The size of the maxima is monotonically increasing with $\varphi_{c}$. For $\varphi_{c}=0$, we have $K_{\mathrm{R}}(\theta)=K_{\mathrm{MN}}$; for $\varphi_{c}=40^{\circ}$, the relative distance of both functions is still less than $0.12 \%$, compare Fig. 7 for the relative difference in terms of the critical friction angle.

\section{Conclusion}

Barodesy is strongly based on Goldscheider's first rule which requires a particular mapping function between stretching directions and stress directions. The cone generated by these stress directions for isochoric stretching represents the critical state surface in the principal stress space. It has been shown that the deviation of this cone from the one defined by the Matsuoka-Nakai criterion is less then $0.12 \%$ for a critical state friction angle of less than $40^{\circ}$. We conclude that this deviation is negligible compared to the uncertainty of the determination of the critical state friction angle. 


\section{References}

[1] J. Desrues, B. Zweschper, and P.A. Vermeer. Database for tests on Hostun RF sand. Institutsbericht 13, Institut für Geotechnik, Universität Stuttgart, 2000 .

[2] W. Fellin and D. Kolymbas. On integrating barodesy for paths starting from zero stress. In S. Pietruszczak and G. Pande, editors, Proceedings of the 2nd International Symposium on Computational Geomechanics, Comgeo II, pages 519-526. International Centre for Computational Engineering (IC2E), 2011.

[3] M. Goldscheider. Grenzbedingung und Fließregel von Sand. Mechanics Research Communication, 3:463-468, 1976.

[4] D. Kolymbas. A generalized hypoelastic constitutive law. In Proc. XI Int. Conf. Soil Mechanics and Foundation Engineering, San Francisco, volume 5, page 2626, Rotterdam, 1985. Balkema.

[5] D. Kolymbas. Sand as an archetypical natural solid. In D. Kolymbas and G. Viggiani, editors, Mechanics of Natural Solids, pages 1-26, 2009.

[6] D. Kolymbas. Barodesy: A new hypoplastic approach. International Journal for Numerical and Analytical Methods in Geomechanics, DOI: 10.1002/nag.1051, 2011.

[7] D. Mašín. A hypoplastic constitutive model for clays. International Journal for Numerical and Analytical Methods in Geomechanics, 29(4):311-336, 2005.

[8] H. Matsuoka and T. Nakai. Stress-deformation and strength characteristics of soil under three different principal stresses. In Proceedings of JSCE 1974, volume 232, pages 59-74, 1974.

[9] D. Muir Wood. Soil behaviour and critical state soil mechanics. Cambridge University Press, 1990.

[10] A. Niemunis and I. Herle. Hypoplastic model for cohesionless soils with elastic strain range. Mechanics of Cohesive-frictional Materials, 2:279-299, 1997. 
[11] P.-A. von Wolffersdorff. A hypoplastic relation for granular materials with a predefined limit state surface. Mechanics of Cohesive-frictional Materials, 1:251-271, 1996. 